\section{Tinea capitis durch Trichophyton violaceum}

Zusammenfassung. Bei einem 13 Jahre alten Mädchen entwickelten sich scheibenförmige Herde mit Haarverlust neben relativ scharf begrenzten Herden mit zum Teil weißlicher pityriasiformer Schuppung und Pustelbildung. Die mykologische Kultur zeigte ein Wachstum von Trichophyton violaceum. Die systemische Behandlung wurde über 6 Wochen mit $100 \mathrm{mg}$ Itraconazol täglich durchgeführt. Zusätzlich kamen lokale Keratolytika und Antibiotika zum Einsatz. Unter dieser Therapie kam es zu einer kompletten Abheilung. T. violaceum führt zu einer endotrichen Infektion und findet sich meist bei Einwanderern aus Nordafrika. Die meist nicht entzündliche Infektion führt zu Haarverlust mit teilweiser Narbenbildung. Die Behandlung mit systemischen Antimykotika ist Therapie der Wahl.

Tinea capitis Caused by Trichophyton Violaceum. A 13year-old girl developed a plaque-like scalp lesion with some hair loss, white pityriasiform desquamation and pustules. Mycological culture showed growth of Trichophyton violaceum. Systemic treatment with itraconazole, $100 \mathrm{mg}$ daily for 6 weeks, as well as topical keratolytic and antibiotic treatment led to complete cure. T. violaceum causes an endotrix infection and is mainly seen in immigrants from Northern Africa. It remains often non-inflammatory, but may cause hair loss which may in part be scarring. Systemic antimycotic treatment is mandatory.

\section{Anamnese}

Seit 9 bis 10 Monaten bestehen bei der Patientin starker Juckreiz mit Rötung und Schuppung sowie Pustelbildung im Nackenhaar. Angeblich sei es auch zu Haarausfall gekommen. Mehrfache Salbenbehandlungen, anfänglich mit Steroiden, später auch Antimykotika, haben zu keiner Besserung geführt.

\section{Befund}

Relativ scharf begrenzte, scheibenförmige, teils entzündlich gerötete und infiltrierte Herde mit deutlicher, teils weißlicher

Akt Dermatol 2001; 27: 34-36

(c) Georg Thieme Verlag Stuttgart · New York ISSN 0340-2541
O. Mainusch ${ }^{1}$, J. Raguz ${ }^{1}$, E. Haneke ${ }^{2}$

${ }^{1}$ Hautklinik Wuppertal, Kliniken der Universität Witten/Herdecke (ehem. Direktor: Prof. Dr. E. Haneke)

2 Klinik Bunæs, Sandvika, Norwegen

(Leiter der dermatol. Abt. Prof. dr. E. Haneke)

pityriasiformer Schuppung neben Alopecia-Herden. Gelegentlich fanden sich auch kleinste Erosionen (Abb.1).

\section{Kultur}

Nach 6 Wochen Wachstum von Trichophyton violaceum Abb. 2).

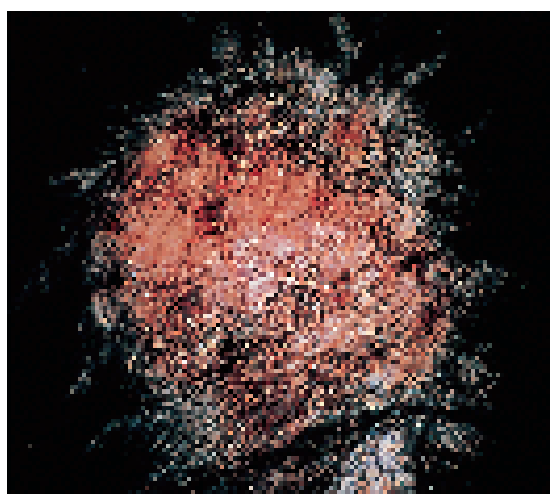

Abb. 1 Klinischer Befund, Kopfhaut.

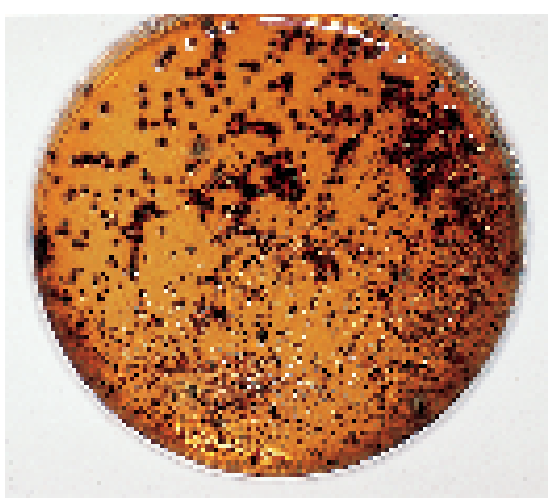

Abb. 2 Kultur, Trichophyton violaceum.

\section{Therapie}

Systemische antimykotische Therapie mit Itraconazol $100 \mathrm{mg} /$ die über 6 Wochen. Zusätzlich lokal keratolytisch und antibiotisch mit Aureomycin-Salbe. Nach 8 Wochen vollständige Abheilung mit Nachwachsen der abgebrochenen Haare. Die Kontrollmykologie war negativ. 


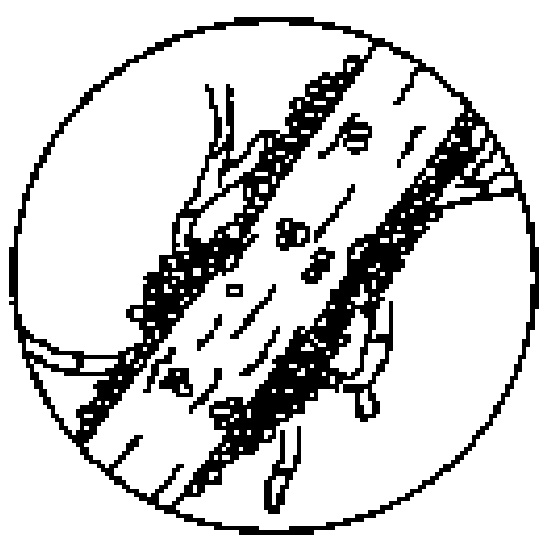

Abb. 3 Ektotriches Pilzwachstum

(Skizze).

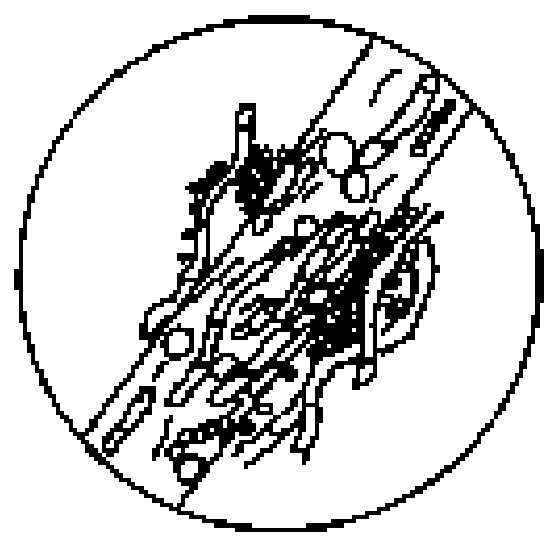

Abb. 4 Endotriches Pilzwachstum (Skizze).

Abb. 5 Trichophyton violaceum (Kultur) (aus Leitfaden der Praktischen Mykologie, Blackwell, Wiss.).

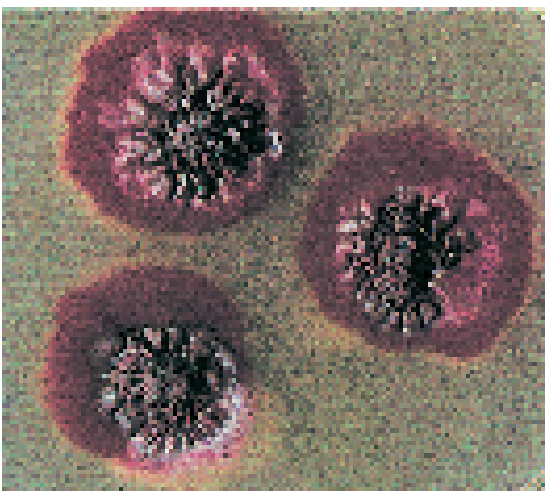

Abb. 6 Trichophyton violaceum

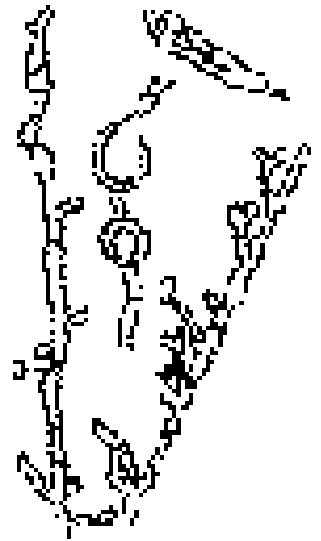
(Mikroskopie, Skizze) (aus Leitfaden der Praktischen Myokologie, Blackwell Wiss.).

\section{Kommentar}

Die Tinea capitis ist eine durch Dermatophyten verursachte Infektion der Haarfollikel der Kopfhaut. Die Dermatophyten dringen entlang den Schäften in die Tiefe und können zu einer entzündlichen oder auch nichtentzündlichen Alopezie führen [6]. Die Infektion resultiert aus der Fähigkeit des Dermatophyten, das Keratin von Haaren, Haut und Nägeln abzubauen [1]. Der bloße Kontakt mit Pilzsporen führt nicht zwangsweise zu einer Infektion, meist sind minimale Traumen der Haut, wie fest geflochtenes Haar, aber auch Abschürfungen und Kratzer, die Voraussetzung. Vor der Pubertät erkrankt das männliche Geschlecht dreimal häufiger als das weibliche. Nach der Pubertät ändert sich das Verhältnis grundlegend. Beim Einsetzen der Pubertät bildet sich die Tinea capitis meist spontan zurück. Nach dem 30. Lebensjahr kommt die Tinea capitis selten vor.

Die drei klinisch bekanntesten Typen der Tinea capitis sind der sog. graue Fleck (grey patch), der schwarze Punkt (black dot) und das Kerion Celsi.

Der graue Fleck entsteht meist durch ektotriche Pilze (Abb. 3), z.B. früher durch Microsporum audouinii, heute, meist reisebedingt durch Microsporum canis. Die Bezeichnung stammt von der gräulichen Haarfarbe, die sich wenige Tage nach der Infektion durch Sporenanlagerung bildet. Die Herde sind umschrieben, dehnen sich aber zentrifugal aus. Am Rand entstehen Pusteln, das Zentrum ist von Schuppen bedeckt. Das Ausmaß der Tinea capitis lässt sich meist erst unter der Woodlampe erkennen.

Der schwarze Punkt bildet das typische Bild der Infektion durch endotriche Pilze (Abb.4) wie Trichophyton tonsurans und Trichophyton violaceum.

Die Infektion ist im Gegensatz zum grauen Fleck nicht immer klinisch auffällig, eine breitflächige Infektion tritt nicht auf. Häufig finden sich einige verstreute schwarze Punkte auf der Kopfhaut durch die direkt an der Kopfhaut abgebrochenen Haare. Eine entzündliche Reaktion besteht nicht. Diese Infektion findet sich auch beim Erwachsenen, der gegenüber einigen Mikrosporumarten eine relative Resistenz besitzt [1]. Hierfür scheint einerseits der Wechsel hin zu gesättigten langkettigen Fettsäuren verantwortlich zu sein, die im Talg enthalten sind und die der Pilz nicht verarbeiten kann, diese werden aber erst nach der Pubertät produziert. Andererseits kommt es zusätzlich zur Rückbildung von Talgdrüsen am Ort der Infektion und somit zu für den Pilz schlechten Wachstumsbedingungen, was die Folge von Spontanheilungen sein kann, so dass ektotriche Infektionen bei Erwachsenen selten sind. Auch scheinen immunologische Faktoren, die mit der T-Zell-vermittelten Immunität assoziiert sind, eine Rolle zu spielen [3].

Das Kerion Celsi ist eine tumorartige Wucherung der Weichteile des Kopfes. Es ist eine Reaktion des Organismus gegen in die Tiefe gelangte Pilzelemente. Kerion Celsi wird meist durch zoophile Dermatophyten ausgelöst.

Nicht unerwähnt sollte jedoch der Favus bleiben, der eine weitere Form der Tinea capitis darstellt. Er wird meist durch Trichophyton schönleinii verursacht, aber auch Trichophyton violaceum kann in seltenen Fällen der Erreger sein. 


\section{Trichophyton violaceum}

\section{Makroskopische Merkmale (Kultur)}

Kulturober- und -unterseite: Violett-dunkelviolett (typische Pigmentbildung).

Cave: Nicht alle Stämme von Trichophyton violaceum (Abb. 5) sind violett, das Spektrum der Farben erstreckt sich von purpur, rot, rosa lavendel bis grau, manche Stämme sind sogar pigmentfrei. Subkulturen werden rasch pleomorph, das heißt, es bildet sich ein weißflammiges Luftmyzel-deshalb immer aus dem vegetativen, violetten Bereich der Kultur überimpfen. Trichophyton violaceum gibt sein Pigment sogar an den Agar ab.

\section{Mikroskopische Merkmale (Abb. 6)}

Mikrokonidien: Sie sind einzellig und eher rar, unter Thiaminzusatz sind sie vermehrt.

Makrokonidien: Sie sind dünnwandig, eher wenige und unterschiedlicher Größe. Sie treten erst spät auf.

Chlamydosporen: Sie sind reichlich vorhanden, interkalar (in den Hyphen).

Hyphen: Die Hyphen wirken knorrig, gestaucht, reichlich septiert und weisen dichotome Verzweigungen wie bei Trichophyton schönleinii auf.

\section{Antimykotische Therapie}

Eine antimykotische Lokaltherapie ist bei der Tinea capitis nicht ausreichend, so dass die orale Gabe systemisch wirksamer Antimykotika, die in die Haarfollikel penetrieren, immer erforderlich sein wird. Neben Griseofulvin, für das über $40 \mathrm{Jah}$ re Erfahrungen bestehen, ist auch Fluconazol zur systemischen Behandlung von Dermatophyteninfektionen in Deutschland zugelassen. Hiermit therapiert werden können auch Kleinkinder über einem Jahr. Der Einsatz von Fluconazol setzt aber voraus, dass keine therapeutische Alternative vorhanden ist. Für das Triazol-Antimykotikum Itraconazol (Sempera ${ }^{\circledR}$ ) zeigten sogar Studien, dass die Wirksamkeit bei Microsporum canis verursachter Tinea capitis gleich gut dem Griseofulvin ist bei besserer Verträglichkeit [2]. Eine ebenso gute Wirkung weist das orale Allylamin Terbinafin (Lamisil ${ }^{\circledR}$ ) auf, was zahlreiche Fallbeschreibungen belegen. Für Trichophyton violaceum und Trichophyton tonsurans fanden sich Heilungsraten von $100 \%$ nach 4 Wochen Therapie mit Terbinafin. Weder Itraconazol noch Terbinafin sind derzeit in Deutschland zur systemischen Therapie bei Kindern zugelassen. Unsere eigenen Erfahrungen sprechen aber bei keinen uns bekannten Nebenwirkungen gegen eine Behandlung bei Kindern [5].

Zusammenfassend ist der Einsatz eines systemischen Antimykotikums bei Tinea capitis zwingend notwendig, da der antimikrobielle Wirkstoff in die Haarfollikel penetrieren muss, was bei topischen Antimykotika nicht ausreichend gewährleistet ist. Eine lokale Behandlung kann zur Reduktion der Schuppung erfolgen, so dass eine Weiterleitung der Infektion verhindert wird.

\section{Literatur}

${ }^{1}$ Elewski BE. Tinea capitis. Dermatol Clinics 1996; 14: 23 - 31

${ }^{2}$ Elewski BE. Tinea capitis: itraconazole in Trichophyton tonsurans infection. J Am Acad Dermatol 1994; 31: 65-67

3 Elewski BE, Hay RJ. International summit on cutaneous antifungal therapy, focus on tinea capitis. Boston, Massachusetts, November 11 - 13, 1994. Ped Dermatol 1996; 13: 69-77

${ }^{4}$ Gianni C, Betti R, Perotta E, Crosti C. Tinea capitis in adults. Mycoses 1995 ; 38: $329-331$

${ }^{5}$ Mainusch O, Hilker O, Haneke E. Onychomycosis in childhood. 8th International Congress Ped Dermatol, Paris 17-20 May, 1998. Ann Dermatol Vénéreol 1998; Suppl: 1557

${ }^{6}$ Nenoff P, Gebauer S, Rytter M, Haustein U-F. Tinea capitis et corporis durch Microsporum canis. Prakt Dermatologie 1997; 3: $302-308$

\section{Dr. Oliver M. Mainusch}

Hautklinik Wuppertal

Zentrum für Dermatologie, Allergologie und Umweltmedizin Klinikum Wuppertal GmbH

Arrenbergerstraße 20-56

42117 Wuppertal

E-mail: Mainusch@klinikum-wuppertal.de 